\title{
Promoting Use of Patient-Centered Health IT: Assessment and Ranking of Incentive Mechanisms
}

\author{
Anton Grube ${ }^{1}$, Tobias Dehling ${ }^{1}$, Kristina Klein ${ }^{2}$, Ali Sunyaev ${ }^{1}$ \\ ${ }^{1}$ Information Systems and Systems Engineering \\ Research Center for IS Design (ITeG) \\ University of Kassel, Germany \\ ${ }^{2}$ Department of Marketing and Brand Management \\ University of Cologne, Germany \\ k.klein@wiso.uni-koeln.de
}

\{grube, tdehling, sunyaev\}@uni-kassel.de

\begin{abstract}
The health care domain is undergoing a sweeping shift from a model of paternalism towards increased patient-centered care. Vendors offering patient-centered health IT use incentive mechanisms to motivate the continued use of health IT. However, incentive mechanisms may not always be beneficial to patientcentered care and may lack focus on actual treatment processes. Therefore, we focus on the research question: What incentive mechanisms are or are not useful for promoting use of patient-centered health IT and why? We assess and rank 28 incentive mechanisms by utility for patient-centered health IT. Findings reveal that reminders and interface improvements are most beneficial and that social comparison and social facilitation mechanisms are most detrimental to patientcentered care. This work extends the scientific knowledge base on patient-centered health IT, establishes a foundation for future research on patient-centered incentive mechanisms, and provides practical audiences with insights on how to effectively design patient-centered health IT.
\end{abstract}

\section{Introduction}

Patient-centered health IT has become an integral part of everyday life. It empowers patients to participate in their own care and exerts a strong influence on patients' health behavior [23, 37, 49]. The increased use of health IT, in particular of patient-centered mobile health IT $[1,13,14]$, such as health management apps on smartphones or tablet PCs, requires however high patient participation. Therefore, high demand exists for incentive mechanisms to motivate patients to start or to continue using health IT. Incentive mechanisms are IT features that persuade users to use IT offerings by appealing to users perception, awareness, attention, or recollection and motivate users to start or proceed using IT [34]. However, most incentive mechanisms are designed to achieve economic goals (eg, motivate users to use profit-based IT) [26, 30]. Application of such incentive mechanisms (eg, monetary, competitive) to promote patient engagement in health care may deter patients from using health IT and may lead to negative effects. Patients may deem certain incentive mechanisms dubious, untrustworthy, or questionable in the health care domain, which has high demands for reliable and serious content. The application of incentive mechanisms that do not evoke trust impedes the empowerment of patients to participate in their own care processes.

Research indicates that application of incentive mechanisms in patient-centered health IT is often based on the needs of care or information system providers rather than the needs of patients $[20,32]$. These kinds of incentive mechanisms in health IT do not improve patient empowerment because it is highly unlikely that patient needs align with the needs of information system or care providers $[12,52]$.

Research on the effectiveness and suitability of incentive mechanisms for patient-centered health IT is sparse $[1,58]$. In particular, research does not offer insights on which incentive mechanisms are suitable for application in health care and how these incentive mechanisms can promote patient-centered care.

In this work, we extend extant research findings through the assessment and ranking of incentive mechanisms for application in patient-centered health IT. We answer the following research questions: (1) Which incentive mechanisms (do not) promote the use of patient-centered health IT? (2) What are the reasons for incentive mechanisms to be beneficial or not? To answer these research questions, we analyze 28 incentive mechanisms, derived from the analysis of persuasive system design elements [34], and assess and rank them by applicability in five domains of patientcentered health care [29].

The remainder of the paper is structured as follows. First, we explain the idea of patient-centered health care and how IT can be useful in this domain. Next, we present the methodology employed in this research and introduce the five dimensions of patient-centered 
care as analysis criteria. We then present our assessment and ranking of the incentive mechanisms. This article ends with a discussion of the findings and resulting conclusions.

\section{Related Research}

The health care domain is undergoing a sweeping shift from paternalism to patient-centered health care $[25,29]$. Patient-centered health care is concerned with care provision consistent with the values, needs, and expectations of patients and is most beneficial when medical professionals involve patients in health care discussions and decisions [13, 29]. Patient-centered health care thus focuses primarily on the wellbeing of the individual patient instead of the financial wellbeing of the overall healthcare industry $[15,27]$. Patient-centeredness implies that actions contributing to health care and treatment processes can also be initiated by patients and decisions are made with patient consultation [17, 54]. Consequently, patients do not feel disregarded and are empowered to have coequal decision-making authority [46]. When patients consult online health IT, they seek for additional information on their treatment (eg, in a case of dissatisfaction with prescribed treatment plans) and expect to find reasonable advice helping to manage their own care [28, 57]. However, patients usually do not have sufficient medical expertise to assess the whole picture of their state of health and to estimate the consequences of their decisions and actions [21]. Hence, medical professionals still must steer patients' decisions and anticipate wrong decisions through additional information provision $[10,21]$.

Patient involvement in treatment processes can occur in different ways $[9,42]$. Patients can provide additional relevant information about their health condition. For example, mobile self-quantification devices can be employed to gather vital signs during daily life activities, or patients can share experiences with a drug or a treatment in respective health communities [21]. Shared information supports the decision process for medical professionals [46]. Patients' experiences offer insights and reveal issues often not considered during conventional medical check-ups [24]. This enhanced information base facilitates longitudinal analyses of patients' state of health by tracking disease courses, symptoms, and recovery processes [38, 54]. This allows patients to recognize potential issues and to contact medical professionals in a timely fashion if needed $[16,42]$. For medical professionals, the information exchange with patients enables new insights into courses of treatment and helps to avoid unforeseen pitfalls with other patients subject to similar diseases or treatment methods [50].
Patient-centered health care represents a radical change in the very traditional health care domain and has the potential to increase patient satisfaction, quality of care, and to improve overall health care outcomes through patient engagement and empowerment $[2,43,51]$, if patients understand the information provided and if they are supported by a supportive health IT landscape $[38,54]$. Health IT that motivates and empowers patients to deal with and comprehend provided information will enable them to take reasonable actions in their own treatment processes. Incentive mechanisms can positively influence patients' motivation and empowerment, but it is not clear which exactly are useful.

\section{Methodology}

\subsection{Research Approach}

Our incentive mechanism assessment is organized in three steps. First, we used the five dimensions (see section 3.2) of the conceptual framework for patientcenteredness [29] as analysis criteria for the patientcentered incentive mechanisms. Second, to obtain a list of incentive mechanisms applicable in IS and IT, we selected 28 incentive mechanisms (see section 4) from the analysis of persuasive system design elements [34]. All other incentive mechanisms that we identified can be mapped on them. We analyzed articles from IS, psychology, and business domains (eg, $[26,55])$ focusing on mechanisms that motivate users to start or to continue using IS and identified independent incentive mechanisms. However, those could always be mapped on one or a combination of persuasive system design elements. For instance, gamification is an independent incentive mechanism that we identified during our research. Gamification is widely employed in IS [55]. It uses persuasive system design elements, such as cooperation, competition, or rewards to create a game-like feeling during app usage and to engage users for a longer period of time in the game. Thus, it is only a special case of a combination of several persuasive system design elements. Third, two independent researchers analyzed the 28 incentive mechanisms through the lens of patient-centered health care and examined if the incentive mechanisms promote, are detrimental to, or are irrelevant for the application in patient-centered health IT and to motivate patient-centered health IT use.

\subsection{Analysis Criteria}

The five core distinguishing factors of patient-centered care (Biopsychosocial Perspective, Patient as a 
Person, Sharing Power and Responsibility, Therapeutic Alliance, and Doctor as a Person) serve as our analysis criteria [29]. Patient-centered health IT should promote or at least not hinder the facilitation of any of these dimensions. We analyze 28 incentive mechanisms (see section 4) by assessing if a mechanism is beneficial, detrimental, or does not affect patient-centered care in any of the five dimensions. In the following, we focus on how health IT should optimally look like, considering each dimension:

Biopsychosocial Perspective (BP): A combination of patients' characteristic and issues (eg, the unwillingness of ostensible 'healthy' hypertension patients to adhere to a treatment plan) beyond biomedical aspects must be considered in the care process [45]. Patient-centered care emphasizes the importance of the biopsychosocial aspects for the success of a medical treatment [19]. Therefore, health IT can only engage patients in the care process if non-medical issues and patients' biological, social, and psychological concerns are considered.

Patient as a Person (PaaP): Since individual patients experience every situation, condition, or illness differently, it is important to consider each patient individually and not to categorize patients, based on symptoms or other superficial aspects [40]. Considering patients as individuals means to understand each patients' story behind an illness and to offer solutions in line with patients' expectations. Hence, health IT that supports patient-centeredness must consider each patient individually and offer individualized treatment alternatives, instead of offering generic solutions based on generalized patient groups.

Sharing Power and Responsibility (PR): Conventional health care can be characterized by an asymmetric relationship between patients and medical professionals, often explained through a "competence gap" [48, p. 162] between medical professionals and patients [39]. The emergence of patient-centered health IT reduces this gap [6] because patients have improved access to health information and are empowered to contribute to their own care process (eg, through health IT). Patient-centered health IT supports certain standards (eg, for information interchange between health IT offerings), informs patients about changes in the care process, and offers the opportunity to criticize (eg, by a feedback option) the care process. Higher patient involvement motivates patients to use IT more frequently, which yields improved effects on patients' individual state of health.

Therapeutic Alliance (TA): The quality of the relationship between medical professionals and patients affects the medical outcomes [15]. Since the effect on medical outcomes is mediated through patients' intention to comply with the proposed treatment plan, an engaging relationship can influence patients' perception of the relevance of the proposed treatment method and improve the willingness to comply with medical professionals' instructions [51]. A trustful, strong, and reliable relationship where patients are involved as equitable members of the decision making and treatment process can motivate patients to take preemptive actions to improve their state of health. Therefore, health IT that supports patient-centered care must foster the perpetuation of the relationship of patients and medical professionals and motivate patients to contribute to the care process.

Doctor as a Person (DaaP): Patient-centered care considers patients and medical professionals as equal participants in the care process with equal rights and obligations concerning the care process. However, medical professionals are better trained and experienced and may have more impact on the care process than patients. Medical professionals' personal qualities, personality, habits, cultural backgrounds, or values also have an impact on the care process $[4,33]$. Therefore, health IT offering patient-centered care must consider medical professionals' influence on both the patients and the care process. Excessive influence of medical professionals may reduce patients' motivation to participate in the care process. Therefore, patient-centered IT must consider medical professionals as experts in the care process, without losing focus on patients' needs and demands.

\subsection{Ranking of Incentive Mechanisms}

Table 1 shows the coding of an incentive mechanism being beneficial for $(+1)$, does not affect $(0)$, or is detrimental to (-1) a dimension of patient-centered health care. Incentive-mechanisms are beneficial (detrimental) for a dimension if they (do not) facilitate patients to receive care or to use any feature of the health IT. In this case, the incentive mechanism will be ranked $+1(-1)$ for the respective dimension of patientcentered health care. If a dimension is not affected at all, the incentive mechanism will be ranked neutral (0) for the respective dimension.

The overall effect of an incentive mechanism is evaluated by taking all five dimensions into account. An incentive mechanism is overall beneficial for patient-centered health care if this mechanism is beneficial for at least one dimension (ranked +1 ) and no other dimension is detrimental (ranked -1). Beneficial mechanisms are likely to motivate patients to use the health IT and improve patient-centered care. Incentive mechanisms are overall detrimental to patient-centered health care if at least one dimension is deemed as detrimental (ranked -1) and no other dimension is deemed as beneficial (ranked +1 ). Detrimental mechanisms do 
Table 1. Ranking Criteria for the Incentive Mechanisms.

\begin{tabular}{|c|c|}
\hline Rank & Description \\
\hline \multicolumn{2}{|r|}{ Biopsychosocial perspective (BP) } \\
\hline+1 & The incentive mechanism promotes the acknowledgement of biological, social and psychological characteristics of patients in health IT. \\
\hline $\mathbf{0}$ & The incentive mechanism has no influence on the acknowledgement of biological, social and psychological characteristics of patients. \\
\hline-1 & The incentive mechanism prevents the acknowledgement of biological, social and psychological characteristics of patients in health IT. \\
\hline \multicolumn{2}{|r|}{ Patient as a person (PaaP) } \\
\hline+1 & The incentive mechanism promotes acknowledgement of patients' expectations and personal characteristics in health IT. \\
\hline $\mathbf{0}$ & The incentive mechanism does not affect the acknowledgement of patients' expectations and personal characteristics in health IT. \\
\hline-1 & $\begin{array}{l}\text { The incentive mechanism supports a classification of the individual patient into generalized groups and rejects the acknowledgement of } \\
\text { individual patient expectations and personal characteristics in health IT. }\end{array}$ \\
\hline \multicolumn{2}{|r|}{ Sharing power and responsibility (PR) } \\
\hline+1 & $\begin{array}{l}\text { The incentive mechanism supports the involvement of patients into the care process and allows to take equitable decisions and neglect } \\
\text { unwanted decisions of medical professionals. }\end{array}$ \\
\hline $\mathbf{0}$ & The incentive mechanism does not affect the balance between patients' and medical professionals' decision power in the care process. \\
\hline-1 & The incentive mechanism restricts patients to make decisions that are crucial to the care process. \\
\hline \multicolumn{2}{|c|}{ Therapeutic alliance (TA) } \\
\hline+1 & The incentive mechanism fosters a high-quality relationship between patients and medical professionals. \\
\hline $\mathbf{0}$ & The incentive mechanism does not affect the relationship of patients and medical professionals at all. \\
\hline-1 & The incentive mechanism prevents patients to build up a qualitative relationship to medical professionals. \\
\hline \multicolumn{2}{|r|}{ Doctor as a person (DaaP) } \\
\hline+1 & $\begin{array}{l}\text { The incentive mechanism acknowledges medical professionals as experts in the care process and allow to apply treatment methods } \\
\text { based on medical professionals' expertise level. }\end{array}$ \\
\hline $\mathbf{0}$ & $\begin{array}{l}\text { The incentive mechanism does not hinder the IT to involve medical professionals according to their expertise level, but the IT at the } \\
\text { same moment does not promote medical professionals' experience and expertise knowledge in the care process. }\end{array}$ \\
\hline-1 & The incentive mechanism prevents medical professionals to influence the care process based on medical professionals' expertise level. \\
\hline
\end{tabular}

not support patient-centeredness at all, or even impede health IT to provide patient-centered health care. Incentive mechanisms are neutral if no dimension of patient-centered health care is deemed beneficial (ranked +1$)$ and no dimension is deemed as detrimental (ranked -1). Neutral incentive mechanisms are irrelevant to promote patient-centeredness and are unlikely to improve patient empowerment. Incentive mechanisms are ranked as discretionary if they are beneficial and detrimental to patient-centered health care at the same time. These mechanisms do not unconditionally support patient-centered care since some of them are detrimental to certain dimensions. However, these mechanisms may still be useful for the application in health IT in general, because the impeded dimension(s) may not be important in this specific case. For instance, workout assistants or calorie counters do not need to support the DaaP dimension, since these kind of health IT usually are not used on behalf of medical professionals.

\section{Influence of Incentive Mechanisms on Patient-Centered Care}

In this work we use the list of 28 incentive mechanisms that are covered in the analysis of persuasive

\footnotetext{
${ }^{1}$ For the readers' convenience, we summarize the influence on the dimensions of patient-centered health care (presented in section 3.2) at the end of each paragraph using the respective abbreviations. The summarized results are presented in Table 2.
}

system design elements [34]. We categorize our results according to the ranking of suitability of the respective mechanism for patient-centered health care (presented in section 3.3).

\subsection{Beneficial Incentive Mechanisms}

Self-monitoring allows patients to track their state of health or the progress towards achieving goals. Selfmonitoring considers patients as individuals demanding control of their care process. Patients can record, manage, and share recorded information to contribute to medical professionals' informational decision base. It fosters the acknowledgement of patients' personal characteristics, provides more information for patients to take decisions, and action in their own health care. Dimensions affected: BP: +1 , PaaP: +1 , SP: +1 , TA: +1, DaaP: $0{ }^{1}$

Tunneling guides patients through a pre-defined (eg, by medical professionals) path in the health IT, to decrease distraction introduced by features unnecessary to achieve a goal. Tunneling mechanisms created and configured by both, medical professionals and patients, can serve as a discussion and decision base to optimize treatment for patients. Conversely, this 
means that tunneling lacks focus on patients' individual preferences. However, doing the same task repeatedly leads to the formation of habits and to higher engagement in the care process. Dimensions affected: BP: 0, PaaP: 0, SP: +1 , TA: +1 , DaaP: +1 .

Reminders are scheduled messages that remind patients to perform a task or to provide feedback information on events, based on patients' individual characteristics. Patients behavior (eg, taking drugs at one time of the day) can be encouraged or strengthened due to development of habits [36]. Dimensions affected: BP: +1 , PaaP: +1 , SP: +1 , TA: +1, DaaP: 0 .

Similarity draws parallels between health IT and patients' personal environments and context. Patients may experience health IT as more convenient if appropriate language and appearance is used (eg, younger patients may see more slang words than mature patients). Similarity refers to patients' cultural and personal characteristics. It addresses patients personally in a meaningful way and motivates them to use the health IT that appeals to their personality. Dimensions affected: BP: +1 , PaaP: +1, SP: 0, TA: 0, DaaP: 0 .

Praising of patients' achievements encourages patients, through positive context-relevant messages, to reinforce an established behavior (eg, daily physical exercises) and health IT use. Health IT may refer to patients' personal biophysical characteristics (eg, praising the amount of steps done). Dimensions affected: BP: +1, PaaP: +1, SP: 0, TA: 0, DaaP: 0 .

Real-world feel incentive mechanisms create a relation between the health IT and the provider offering the health IT, which leads to an improved perception of seriousness of the content provided and a stronger relation to patients therapy, biopsychosocial characteristics, or health condition can be created. Patients' expectations for real outcomes (eg, better health condition) lead to real intention to use health IT. Dimensions affected: BP: +1 , PaaP: 0, SP: +1 , TA: +1 , DaaP: 0 .

Simulation enables patients to see the outcome of certain health IT features and an interconnection of cause and effect. For instance, a smoking cessation health IT might illustrate the money amount saved [56], which leads to the visualization of effects (saved money in future) caused by a specific action (stop smoking now). Patients recognize the goal and are motivated to adhere to treatment plans. Dimensions affected: BP: 0, PaaP: 0, SP: +1 , TA: 0, DaaP: +1 .

Social learning implies that patients learn from other patients' behavior through passive observation (eg, patients with similar symptoms or treatment progress). In collaboration with medical professionals, adjustments can be made to patients' treatment process, raising patients' decision power and the therapeutic alliance. Dimensions affected: BP: +1 , PaaP: 0 , SP: +1, TA: +1 DaaP: 0 .
Personalization adapts health IT content to patients' personal characteristics (eg, name) and creates a reference to patients' personality. This leads to higher motivation to use the health IT through a perceived connection to patients' personal condition [44]. Personalization integrates patients' biopsychosocial characteristics (eg, previously recorded and stored in a personal account) in the health IT to appeal to patients' personality. Dimensions affected: BP: +1 , PaaP: +1 , SP: 0, TA: 0, DaaP: 0.

Reduction mechanisms reduce complex sequences of tasks to a simple task that helps patients to overview necessary steps to achieve a goal. Reduced complexity leads to less cognitive effort of patients while using the health IT, which in turn leads to higher perceived ease of use. Health IT considers patients' individual characteristics for reduced task complexity but also medical professionals' expertise in the treatment process (eg, defining steps to fulfil a task), which leads to shared decision making. Continuous exchange of feedback between both, the patients' and medical professionals, can lead to a satisfactory solution for both. Dimensions affected: BP: +1 , PaaP: +1 , SP: +1 , TA: +1 , DaaP: 0.

Rehearsal are samples of features that patients can try to experience the effect (or outcome) of actions. Patients can execute a function of the health IT with fictional information (eg, a demonstration profile) without setup effort. Trying features of the health IT allows users to decide if a feature is relevant and necessary, giving them decision making power. Dimensions affected: BP: 0, PaaP: 0, SP: +1, TA: 0, DaaP: 0.

\subsection{Discretionary Incentive Mechanisms}

Trustworthiness raises users trust in health IT content through the reduction of potential sources of mistrust (eg, providing information on organizations with access to data, stored in the health IT offering). This leads to higher intention to use health IT and participate in the care process [18]. However, overemphasizing on health IT for health care may deprive power of medical professionals' role in the care process, which may disturb the relationship of patients and medical professionals. In return, patients win on decision power. Dimensions affected: BP: 0, PaaP: 0, SP: +1 , TA: 0, DaaP: -1.

Suggestions are messages (referring to patients biopsychosocial and personal characteristics) with specific instructions for patients that are intended to assist patients during the use of health IT. Suggestions may lead patients through a path of steps that are needed to fulfil a task, or recommend alternative actions or treatment steps (eg, alternative diet plans), which may de- 
Table 2. Ranking of Incentive Mechanisms for Patient-Centered Health Care.

\begin{tabular}{|c|c|c|c|c|c|c|}
\hline R. & Incentive mechanism & BP & PaaP & SP & TA & DaaP \\
\hline \multirow{11}{*}{ 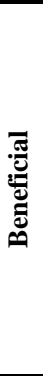 } & Self-monitoring & +1 & +1 & +1 & +1 & 0 \\
\hline & Tunneling & 0 & 0 & +1 & +1 & +1 \\
\hline & Reminders & +1 & +1 & +1 & +1 & 0 \\
\hline & Similarity & +1 & +1 & 0 & 0 & 0 \\
\hline & Praise & +1 & +1 & 0 & 0 & 0 \\
\hline & Real-world feel & +1 & 0 & +1 & +1 & 0 \\
\hline & Simulation & 0 & 0 & +1 & 0 & +1 \\
\hline & Social learning & +1 & 0 & +1 & +1 & 0 \\
\hline & Personalization & +1 & +1 & 0 & 0 & 0 \\
\hline & Reduction & +1 & +1 & +1 & +1 & 0 \\
\hline & Rehearsal & 0 & 0 & +1 & 0 & 0 \\
\hline \multirow{10}{*}{ 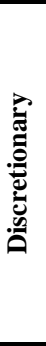 } & Trustworthiness & 0 & 0 & +1 & 0 & -1 \\
\hline & Suggestion & +1 & +1 & +1 & -1 & -1 \\
\hline & Tailoring & +1 & +1 & 0 & 0 & -1 \\
\hline & Authority & -1 & -1 & +1 & -1 & -1 \\
\hline & Rewards & +1 & +1 & -1 & -1 & -1 \\
\hline & Verifiability & 0 & 0 & +1 & 0 & -1 \\
\hline & Normative influence & 0 & -1 & -1 & 0 & +1 \\
\hline & Recognition & -1 & -1 & +1 & 0 & -1 \\
\hline & 3rd party endorsement & 0 & 0 & +1 & 0 & -1 \\
\hline & Cooperation & +1 & 0 & +1 & -1 & -1 \\
\hline$\dot{\mathbf{z}}$ & Liking & 0 & 0 & 0 & 0 & 0 \\
\hline \multirow{6}{*}{ 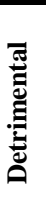 } & Surface credibility & $\overline{0}$ & 0 & 0 & 0 & -1 \\
\hline & Social role & 0 & 0 & 0 & -1 & -1 \\
\hline & Expertise & 0 & 0 & 0 & -1 & -1 \\
\hline & Competition & -1 & -1 & 0 & 0 & -1 \\
\hline & Social comparison & -1 & -1 & 0 & -1 & -1 \\
\hline & Social facilitation & -1 & -1 & -1 & -1 & -1 \\
\hline
\end{tabular}

+1 = Beneficial; 0, N. = Neutral; -1 = Detrimental; R. = Rank

crease medical professionals' influence on the treatment process. Dimensions affected: BP: +1 , PaaP: +1 , SP: +1 , TA: -1 , DaaP: -1 .

Tailoring mechanisms adopt health IT content to patients' personal characteristics providing only relevant and interesting information to the patient [46]. Tailoring mechanisms offer medical professionals possibilities to adapt a treatment to patients needs and to guide patients through the care process. However, patients might use tailoring mechanisms to tailor their health IT according to their needs and demands, avoiding unpleasant instructions (eg, regarding diet or physical activities) and thus undermine medical professionals' authority. Additionally, health IT can propose content contrary to medical professionals' instructions. Dimensions affected: BP: +1 , PaaP: +1 , SP: 0 , TA: 0, DaaP: -1 .

Authority of health IT content can be achieved by referring to authorities in the specific domain while presenting the content, which strengthens patients' decision power in the care process. However, patientcentered health IT should always provide reliable and correct content, verified by different authority levels and instances. Therefore, this incentive mechanism is capable to question medical professionals' authority position. Furthermore, health IT may gain authority over medical professionals and lose focus on patients' objectives. Dimensions affected: BP: -1, PaaP: -1, SP: +1, TA: -1 , DaaP: -1 .

Rewards is an incentive mechanism to acknowledge patients' efforts in the care process. Health IT may acknowledge social, physical, or medical efforts of patients and award patients with virtual rewards (eg, badges, medals). In turn, this might lead to patients' focusing on getting the reward instead of complying with the treatment plan. Dimensions affected: BP: +1 , PaaP: +1 , SP: -1 , TA: -1 , DaaP: -1 .

Verifiability offers patients the possibility to validate content provided in the health IT (eg, by comparing it to medical professionals' instructions). This can obviously strengthen the relationship between patients and medical professionals, but also impede the introduction of treatment methods that are not verifiable by health IT. Furthermore, health IT can transform to verification tools for medical professionals' instructions, that may weaken medical professionals' decision and expertise power. Dimensions affected: BP: 0, PaaP: 0, SP: +1 , TA: 0, DaaP: -1 .

Normative influence exerts pressure on patients through social (or legal) norms. Patients find themselves forced to comply with these norms (eg, average weight or BMI of other patients). However, alignment with social norms leads to lost-in-the-masses patients, which is detrimental to the PaaP and BP dimensions. However, medical professionals can exert additional influence through generally acknowledged norms, to persuade patients to comply with medical professionals' instructions. Dimensions affected: BP: 0, PaaP: 1, SP: -1, TA: 0, DaaP: +1

Recognition of individual achievements (eg, by like-minded patients, medical professionals, or close relatives) motivates patients to proceed to use health IT if their efforts are honored (eg, in form of public praise). Therefore, patients may feel encouraged to receive further recognition for their efforts and lose focus on their actual goal, the personal state of health. Dimensions affected: BP: -1 , PaaP: -1 , SP: +1 , TA: 0 , DaaP: -1

3rd-party endorsement improves the verifiability of health IT content by authorized providers (eg, certification authorities [53]). Patients can rely on certifications of health IT, which leads to less effort to verify health IT content personally and improve the decision base. However, 3rd-party endorsement may act as controlling instance between patients and medical professionals and lead to an imbalance in the patient-medical professional relationship and reduce medical professionals' authority. Dimensions affected: BP: 0, PaaP: 0, SP: +1 , TA: 0, DaaP: -1 . 
Cooperation creates a shared goal that patients want to achieve together. Based on biophysical characteristics, patients can achieve comparable goals (eg, two obese patients may be challenged to together lose four pounds per week). However, patients focusing on common goals might be distanced from medical professionals' goals (eg, loose more weight than necessary). Dimensions affected: BP: +1 , PaaP: $0, \mathrm{SP}:+1$, TA: -1 , DaaP: -1 .

\subsection{Neutral Incentive Mechanism}

Liking influences patients' perception of health IT through visually appealing elements. Patients are more likely to use health IT features if they like the appearance of the health IT. Literature supports the positive influence of visual appealing interfaces on use behavior [7]. However, patients' attention may be distracted from relevant health IT features, which neutralizes the effect on patient-centered care: BP: 0, PaaP: 0, SP: 0, TA: 0, DaaP: 0.

\subsection{Detrimental Incentive Mechanisms}

Surface credibility refers to a convenient look-andfeel of health IT. An advantageous surface may lead to premature expectations about the health IT before patients even perform the first task. However, health IT (eg, for the support of complex treatments) with complex interfaces may discourage patients from using the health IT [11], albeit medical professionals recommending to do so. Dimensions affected: BP: 0, PaaP: 0, SP: 0, TA: 0, DaaP: -1 .

Social role of health IT requires health IT to play a particular role in patients' care process (eg, educational, controlling, or assisting). Patients know what they expect from health IT (eg, advice on a treatment plan) and refer to health IT. This weakens medical professionals' authority and relationship. Dimensions affected: BP: 0, PaaP: 0, SP: 0, TA: -1 , DaaP: -1 .

Expertise levels of provided content in health IT have a large influence on patients' usage intention, if patients perceive a high level of expertise. However, health IT may be perceived too competent and reduce medical professionals' influence in the care process, which disturbs the relationship or undermines medical professionals' expertise level. Dimensions affected: BP: 0, PaaP: 0, SP: 0, TA: -1 , DaaP: -1 .

Competition motivates patients to contribute to the health care process based on other patients' level of activity. Patients may be engaged in a game (eg, to burn a certain amount of calories in one month), which fosters competitive behavior of patients, which leads to higher engagement. Since competition mechanisms focus on other patients' personal characteristics to compete, patients' biophysical and personal characteristics are forced into the background. Focus on competition may make patients lose focus on medical professionals' instructions. Dimensions affected: BP: -1 , PaaP: -1, SP: 0, TA: 0, DaaP: -1 .

Social comparison motivates patients to use health IT through comparison to other patients' performance (eg, goal achievement). Patients are more motivated to perform better in the next comparison to other patients. Hence, patients can see if they execute tasks and actions efficiently or not (eg, do not lose enough weight). This may lead to lost-in-the-masses patients, assessed by mass average, and rejection of the $\mathrm{PaaP}$ and $\mathrm{BP}$ dimensions. Furthermore, this might lead to a weak relationship and hence to less influence by medical professionals. Dimensions affected: BP: -1 , PaaP: -1 , SP: 0, TA: -1 , DaaP: -1 .

Social facilitation motivates users to use health IT because like-minded patients do the same. Higher perceived empathy or social support by other patients [31] lead to lost-in-the-masses patients, that follow the actions of other patients (rather than rely on medical professionals' instructions) without a clear understanding of purpose, medical necessity, and boundary conditions. Dimensions affected: BP: -1 , PaaP: -1 , SP: -1 , TA: -1 , DaaP: -1 .

\section{Discussion}

This work assesses and ranks incentive mechanism for patient-centered health IT. Building on our exploratory analysis of incentive mechanisms with respect to patient-centered health care dimensions, we assess which incentive mechanisms are beneficial or detrimental to patient-centered health care.

Despite the importance of medical professionals in the care process, our research findings indicate that most of the analyzed incentive mechanisms are detrimental to the equitable involvement of medical professionals in patient-centered health IT environments (see Table 2; column 'DaaP'). Overall, incentive mechanisms are rather focusing on patients' needs and demands reducing the focus on the medical necessity of the offered features. Four out of six detrimental incentive mechanisms focus on social support for patients (social role, competition, social comparison, and social facilitation; see Table 2, row 'Detrimental'). These improve social influence but reduce influence of medical professionals on the treatment process. Extant research indicates a strong demand of patients [8] for social interaction features in health IT with both, other patients and medical professionals [5]. Various reasons for social support or social information seeking from the patients' side exist: Patients might experience dissatisfaction or comprehension issues with 
the information provided during the care process [41]. Medical professionals often are not able to provide sufficient care to patients in practice or inpatient treatment due to a lack of resources [47]. Social interaction through health IT requires additional resources (which might not be available) and can lead to decreasing standards of care. Furthermore, often, legal restrictions forbid online treatment. (eg, Germany [35]) or insurance companies refuse to pay for online treatment services [22], which impedes the application of incentive mechanisms that allow medical professionals to provide online treatment. Overall, only normative influence, simulation and tunneling (see Table 2, column 'DaaP') are beneficial for active involvement of medical professionals in the patient-centered care process. Therefore, patient-centered incentive mechanisms convert medical professionals' role in the care process into observational or recommending positions. Due to a strong focus of incentive mechanisms on equipping patients with grater negotiation and decision power (see Table 2, column 'SP' and 'TA'), the influence of medical professionals on the care process shrinks, entailing drainage of medical expertise out of the care process and increasing the risk of medication errors. Patients must indisputable receive information empowering them to take informed decisions concerning their care process, but many decisions must be discussed with medical professionals to prevent harmful consequences to patients' state of health [21].

Further findings indicate that no incentive mechanism is beneficial for the patient-centered care in all five dimensions; only liking is fully neutral for patientcenteredness, and only social facilitation is detrimental in all five dimensions (see Table 2). Therefore, to achieve support of patient-centered health care in all dimensions, it is necessary to use a combination of incentive mechanisms, depending on the field of application and the objective of the health IT. For instance, health IT that aims to provide treatment-related information for patients may use a combination of normative influence, cooperation, real-world feel, and personalization. This combination facilitates information provision, appeals to patients needs and demands, enables social exchange to better understand the provided information, involves medical professionals in the information provision process, supports medical professionals' instructions with common norms, and creates a relation to real-world outcomes. In turn, the imprudent application of one unsupportive incentive mechanism may be detrimental for the patient-centeredness of health IT. For instance, the application of the incentive mechanism verifiability improves patients' negotiation and decision-making power through the possibility to verify incomprehensible information or treatment instructions, but might lead to non-adherence to medical professionals' instructions.

This work has the following limitations. First, incentive mechanisms analyzed in this work are based on persuasive system design elements, which might ignore further incentive mechanisms that come from other domains (eg, IS continuance [3]) or from recommendations of medical professionals. Considering further incentive mechanisms might extend the scope of analysis, and the amount and quality of patient-centered incentive mechanisms. Second, we assessed the eligibility of the incentive mechanisms by the application of five dimensions of patient-centered health care. An empirical evaluation of incentive mechanisms might reveal different effects on engagement and empowerment of patients in the treatment process, that differ from their respective effect on patient-centered health care dimensions.

To assess incentive mechanisms' influence on patients' perception of patient-centeredness, it is necessary to develop and evaluate patient-centered health IT in clinical environments. The results might shed light on the motivational power of individual incentive mechanisms and identify inefficient mechanisms, that are detrimental to patient-centered health care. Future studies might investigate the application of incentive mechanisms in a real treatment process or focus on differences by application area. Furthermore, future studies might investigate if a combined application of incentive mechanisms leads to synergy effects. For instance, the application of liking alone might not affect any dimension of patient-centered health care. However, liking in combination with surface credibility might lead to user interfaces capable to motivate health IT use through convenient and convincing design. It is also important to analyze incentive mechanisms used in the current health IT landscape. Qualitative and empirical analyses of existing patient-centered health IT solutions might reveal incentive mechanisms not analyzed in this work or disclose efficient combinations of common incentive mechanisms in health-care environments. Although incentive mechanisms are tested for efficacy in other domains, the application in patient-centered health IT is not evaluated so far. Further research might include the analysis of incentive mechanisms in clinical trials and reveal a relation to improved clinical outcomes.

Our study contributes to scientific knowledge base in multiple ways. First, by assessing incentive mechanisms for utility in patient-centered health care, we direct attention to a promising mechanism to improve health IT use and medical outcomes, without neglecting the idea of patient-centered health care. Our results indicate that the application of incentive mechanisms 
can be context-sensitive and can diminish the advantages of patient-centered health care if applied haphazardly. Second, by identifying, assessing, and ranking these incentive mechanisms, we provide foundations for future research on patient-centered incentive mechanisms. Although research on incentive-mechanisms already exists in various domains, none of them analyzes incentive mechanisms in patient-centered health IT contexts or propose a patient-centered perspective on incentive mechanisms for health IT to the best of our knowledge [58]. Based on our exploratory study and extant research on incentive mechanisms, we propose a classification for patient-centered incentive mechanisms for patient-centered health IT. Third,

\section{References}

[1] Bates, D.W. and A. Bitton, "The Future of Health Information Technology in the Patient-Centered Medical Home", Health Affair, 29(4), 2010, pp. 614-622.

[2] Bertakis, K.D. and R. Azari, "Patient-Centered Care is Associated with Decreased Health Care Utilization", J Am Board Fam Med, 24(3), 2011, pp. 229-239.

[3] Bhattacherjee, A., "Understanding Information Systems Continuance", MIS Quart, 25(3), 2001, p. 351.

[4] Borrell-Carrio, F., "Preventing Errors in Clinical Practice: A Call for Self-Awareness", Ann Fam Med, 2(4), 2004, pp. 310-316.

[5] Bosslet, G.T.; A.M. Torke; S.E. Hickman; C.L. Terry; and P.R. Helft, "The Patient-Doctor Relationship and Online Social Networks: Results of a National Survey", J Gen Intern Med, 26(10), 2011, pp. 1168-1174.

[6] Byrne, P. and B. Long, Doctors Talking to Patients., HMSO, London, UK, 1976.

[7] Caine, K.; S. Kohn; C. Lawrence; R. Hanania; E. Meslin; and W. Tierney, "Designing a Patient-Centered User Interface for Access Decisions about EHR Data", J Gen Intern Med, 30(1), 2015, pp. 7-16.

[8] Centola, D., "Social Media and the Science of Health Behavior", Circulation, 127(21), 2013, pp. 2135-2144.

[9] Coulter, A. and J. Ellins, "Effectiveness of Strategies for Informing Educating and Involving Patients", Brit Med J, 335(7609), 2007, pp. 24-27.

[10] Davies, P., "Should Patients be Able to Control Their Own Records?", Brit Med J, 345, 2012, e4905.

[11] Davis, F.D., "User Acceptance of Information Technology", Int J Man Mach Stud, 38(3), 1993, pp. 475-487.

[12] Dayer, L.; S. Heldenbrand; P. Anderson; P.O. Gubbins; and B.C. Martin, "Smartphone Medication Adherence Apps: Potential Benefits to Patients and Providers", J Am Pharm Assoc, 53(2), 2013, pp. 172-181.

[13] Dehling, T. and A. Sunyaev, "Secure Provision of Patient-Centered Health Information Technology Services in Public Networks", Electron Mark, 24(2), 2014, pp. 89-99. practitioners can use our results to develop more focused health IT applications for patients and to identify most suitable incentive mechanisms for a particular scope and objective of patient-centered health IT. Health IT vendors might use the incentive mechanisms identified to implement these in 'ready-to-use' health IT offerings to promote patient involvement, participation and empowerment in the care process. Design and development of health IT appealing to patients' desires and beliefs might lead to more frequent health IT use. This may in turn lead to higher effectiveness of these health IT offering and improve health care outcomes.

[14] Demiris, G.; L.B. Afrin; S. Speedie; K.L. Courtney; M. Sondhi; V. Vimarlund; C. Lovis; W. Goossen; and C. Lynch, "Patient-Centered Applications: Use of Information Technology to Promote Disease Management and Wellness. A White Paper by the AMIA Knowledge in Motion Working Group", J Am Med Inform Assn, 15(1), 2008, pp. 8-13.

[15] Epstein, R.M.; P. Franks; K. Fiscella; C.G. Shields; S.C. Meldrum; R.L. Kravitz; and P.R. Duberstein, "Measuring Patient-Centered Communication in Patient-Physician Consultations", Soc Sci Med, 61(7), 2005, pp. 1516-1528.

[16] Eysenbach, G., "Consumer Health Informatics", Brit Med J, 320(7251), 2000, pp. 1713-1716.

[17] Eysenbach, G., "Medicine 2.0: Social Networking, Collaboration, Participation, Apomediation, and Openness", J Med Internet Res, 10(3), 2008, e22.

[18] Gefen, D.; E. Karahanna; and D.W. Straub, "Trust and TAM in Online Shopping: An Integrated Model", MIS Quart, 27(1), 2003, pp. 51-90.

[19] Grol, R.; J. de Maeseneer; M. Whitfield; and H. Mokkink, "Disease-Centred versus Patient-Centred Attitudes: Comparison of General Practitioners in Belgium, Britain and The Netherlands", Fam Pract, 7(2), 1990, pp. 100-103.

[20] Grube, A.; T. Dehling; and A. Sunyaev, "How Do Patients Expect Apps to Provide Drug Information?", in Proceedings of the 50th HICSS, HI, USA, January 4-7, 2017.

[21] Hamilton, D.W., "Shared Decision Making Asks Patients to Share their Aims and Values for Treatment", Brit Med J, 348, 2014, g1435.

[22] Hawn, C., "Take two Aspirin and Tweet me in the Morning: How Twitter, Facebook, and other Social Media are Reshaping Health Care", Health Affair, 28(2), 2009, pp. 361-368.

[23] Hibbard, J.H. and J. Greene, "What the Evidence Shows about Patient Activation: Better Health Outcomes and Care Experiences; Fewer Data on Costs", Health Affair, 32(2), 2013, pp. 207-214.

[24] Horwitz, R.I.; M.R. Cullen; J. Abell; and J.B. Christian, "(De)Personalized Medicine", Science, 339(6124), 2013, pp. 1155-1156. 
[25] Kaba, R. and S. Prasanna, "The Evolution of the Doctor-Patient Relationship", Int J Surg, 5(1), 2007, pp. 57-65.

[26] Kauffman, R.J. and P.P. Tallon, Economics, Information Systems, and Electronic Commerce: Empirical Research, Taylor \& Francis, 2014.

[27] Kleinke, J.D., "Dot-Gov: Market Failure and the Creation of a National Health Information Technology System", Health Affair, 24(5), 2005, pp. 1246-1262.

[28] Li, N.; S. Orrange; R.L. Kravitz; and R.A. Bell, "Reasons for and Predictors of Patients' Online Health Information Seeking Following a Medical Appointment", Fam Pract, 31(5), 2014, pp. 550-556.

[29] Mead, N. and P. Bower, "Patient-Centredness: A Conceptual Framework and Review of the Empirical Literature", Soc Sci Med, 51(7), 2000, pp. 1087-1110.

[30] Meng, X. and B. Gallagher, "The Impact of Incentive Mechanisms on Project Performance", Int J Proj Manag, 30(3), 2012, pp. 352-362.

[31] Nambisan, P., "Information Seeking and Social Support in Online Health Communities: Impact on Patients' Perceived Empathy", J Am Med Inform Assn, 18(3), 2011, pp. 298-304.

[32] Nicolas-Rocca, T.S.; B. Schooley; and S.-J. Joo, "Design and Development of a Patient-Centered E-Health System to Improve Patient Understanding at Discharge", Commun AIS, 34(1), 2014, pp. 453-476.

[33] Novack, D.H.; A.A. Suchman; W. Clark; R.M. Epstein; E. Najberg; and C. Kaplan, "Calibrating the Physician: Personal Awareness and Effective Patient Care", J Amer Med Assoc, 278(6), 1997, pp. 502-509.

[34] Oinas-Kukkonen, H. and M. Harjumaa, "Persuasive Systems Design: Key Issues, Process Model, and System Features", Commun AIS, 24(1), 2009, pp. 485-500.

[35] OsborneClarke, Between Consulting and Treatment: Telemedicine in Germany is Still Hampered by Legal Barriers. 2017. http://www.osborneclarke.com/insights/consulting-treatment-telemedicine-germany, archived at: webcitation.org/6tRVxDFE7, accessed 6-15-2017.

[36] Oulasvirta, A.; T. Rattenbury; L. Ma; and E. Raita, "Habits Make Smartphone Use more Pervasive", Pers Ubiquit Comput, 16(1), 2012, pp. 105-114.

[37] Ozdalga, E.; A. Ozdalga; and N. Ahuja, "The Smartphone in Medicine: A Review of Current and Potential Use Among Physicians and Students", J Med Internet Res, 14(5), 2012, e128.

[38] Parish, E., "BMJ Roundtable Debate: How Can we get Better at Providing Patient Centred Care?", Brit Med J, 350, 2015, h412.

[39] Parsons, T., The Social System, The Free Press, Glencoe, IL (USA), 1951.

[40] Petrie, K.J. and J. Weinman, "Patients' Perceptions of Their Illness", Curr Dir Psychol Sci, 21(1), 2012, pp. 60-65.

[41] Rajasundaram, R.; S. Phillips; and N.R. Clay, "Information Leaflet Used in Out-Patient Clinics: A Survey of Attitude and Understanding of the User", Int J Health Care Qual Assur, 19(6-7), 2006, pp. 575-579.
[42] Ricciardi, L.; F. Mostashari; J. Murphy; J.G. Daniel; and E.P. Siminerio, "A National Action Plan to Support Consumer Engagement via E-Health", Health Affair, 32(2), 2013, pp. 376-384.

[43] Richards, T.; A. Coulter; and P. Wicks, "Time to Deliver Patient Centred Care", Brit Med J, 350, 2015, h530.

[44] Salonen, V. and H. Karjaluoto, "Web personalization: The State of the Art and Future Avenues for Research and Practice", Telemat Inform, 33(4), 2016, pp. 1088-1104.

[45] Schofield, T., "Patient-Centered Medicine: Transforming the Clinical Method", Brit Med J, 311(1580), 1995.

[46] Shaw, J. and M. Baker, "“Expert Patient" - Dream or Nightmare?", Brit Med J, 328(7442), 2004, pp. 723-724.

[47] Shirom, A.; N. Nirel; and A.D. Vinokur, "Overload, Autonomy, and Burnout as Predictors of Physicians' Quality of Care", J Occup Health Psych, 11(4), 2006, pp. 328-342.

[48] Silverman, J.; S. Kurtz; and J. Draper, Skills for Communicating with Patients, 3rd edn., CRC Press, 2016.

[49] Smith, S.P. and A.C. Barefield, "Patients Meet Technology: The Newest in Patient-Centered Care Initiatives", Health Care Manage R, 26(4), 2007, pp. 354-362.

[50] Stacey, D.; F. Légaré; K. Lewis; M.J. Barry; C.L. Bennett; K.B. Eden; M. Holmes-Rovner; H. Llewellyn-Thomas; A. Lyddiatt; R. Thomson; and L. Trevena, "Decision Aids for People Facing Health Treatment or Screening Decisions", Cochrane Db Syst Rev, 2017(4), 2017.

[51] Stewart, M.; J.B. Brown; A. Donner; I.R. McWhinney; J. Oates; W.W. Weston; and J. Jordan, "The Impact of Patient-Centered Care on Outcomes", J Fam Pract, 49(9), 2000, pp. 796-804.

[52] Sunyaev, A.; D. Chornyi; C. Mauro; and H. Krcmar, "Evaluation Framework for Personal Health Records: Microsoft HealthVault Vs. Google Health", in Proceedings of the 43rd HICSS, Hi, USA, January 05-08, 2010.

[53] Sunyaev, A. and S. Schneider, "Cloud Services Certification", Commun ACM, 56(2), 2013, pp. 33-36.

[54] Swan, M., "Health 2050: The Realization of Personalized Medicine Through Crowdsourcing, the Quantified Self, and the Participatory Biocitizen", J Pers Med, 2(3), 2012, pp. 93-118.

[55] Thiebes, S.; S. Lins; and D. Basten, "Gamifying Information Systems - A Synthesis of Gamification Mechanics and Dynamics", in Proceedings of the ECIS 2014, Tel Aviv, Israel, June 9-11, 2014.

[56] Webb, M.S.; V.N. Simmons; and T.H. Brandon, "Tailored Interventions for Motivating Smoking Cessation: Using Placebo Tailoring to Examine the Influence of Expectancies and Personalization", Health Psychol, 24(2), 2005, pp. 179-188.

[57] Wilkins, A.S., "Expanding Internet Access for Health Care Consumers", Health Care Manage R, 24(3), 1999, pp. $30-41$.

[58] Win, K.T.; J. Mullan; S. Howard; and H. Oinas-Kukkonen, "Persuasive Systems Design features in Promoting Medication Management for consumers", in Proceedings of the 50th HICSS, HI, USA, January 4-7, 2017. 